See discussions, stats, and author profiles for this publication at: https://www.researchgate.net/publication/332386836

\title{
Reproducibility and Repeatability of Five Different Technologies for Bar Velocity Measurement in Resistance Training
}

Article in Annals of Biomedical Engineering • April 2019

DOI: 10.1007/s10439-019-02265-6

CITATIONS

15

7 authors, including:

A Javier Courel Ibáñez

University of Murcia

82 PUBLICATIONS 400 CITATIONS

SEE PROFILE

Ricardo Morán-Navarro

University of Murcia

31 PUBliCATIONS 325 CITATIONS

SEE PROFILE
READS

899

Alejandro Martínez Cava

University of Murcia

17 PUBLICATIONS 62 CITATIONS

SEE PROFILE

(7) Pablo Escribano

University of Murcia

1 PUBLICATION 14 CITATIONS

SEE PROFILE

Some of the authors of this publication are also working on these related projects:

Project Effects of low-load and low-volume resistance training on physical performance in young and adult soccer players View project

Project MLSS economic and valid determination procedure in cyclists View project 


\title{
Reproducibility and Repeatability of Five Different Technologies for Bar Velocity Measurement in Resistance Training
}

\author{
Javier Courel-Ibáñez, ${ }^{1}$ Alejandro Martínez-Cava, ${ }^{1}$ Ricardo Morán-Navarro, ${ }^{1}$ \\ Pablo Escribano-Peñas, ${ }^{1}$ Javier Chavarren-Cabrero, ${ }^{2}$ Juan José González-Badillo, ${ }^{3}$ \\ and Jesús G. Pallarés (iD ${ }^{1}$ \\ ${ }^{1}$ Human Performance and Sports Science Laboratory, Faculty of Sport Sciences, University of Murcia, C/ Argentina s/n, \\ Santiago de la Ribera, Murcia, Spain; ${ }^{2}$ Department of Physical Education, University of Las Palmas de Gran Canaria, Las \\ Palmas de Gran Canaria, Spain; and ${ }^{3}$ Faculty of Sport, Pablo de Olavide University, Seville, Spain
}

(Received 19 January 2019; accepted 5 April 2019)

Associate Editor Stefan M. Duma oversaw the review of this article.

\begin{abstract}
This study aimed to analyze the agreement between five bar velocity monitoring devices, currently used in resistance training, to determine the most reliable device based on reproducibility (between-device agreement for a given trial) and repeatability (between-trial variation for each device). Seventeen resistance-trained men performed duplicate trials against seven increasing loads (20-30-40-50-60-70$80 \mathrm{~kg}$ ) while obtaining mean, mean propulsive and peak velocity outcomes in the bench press, full squat and prone bench pull exercises. Measurements were simultaneously registered by two linear velocity transducers (LVT), two linear position transducers (LPT), two optoelectronic camera-based systems (OEC), two smartphone video-based systems (VBS) and one accelerometer (ACC). A comprehensive set of statistics for assessing reliability was used. Magnitude of errors was reported both in absolute $\left(\mathrm{m} \mathrm{s}^{-1}\right)$ and relative terms $(\% 1 \mathrm{RM})$, and included the smallest detectable change (SDC) and maximum errors (MaxError). LVT was the most reliable and sensitive device (SDC 0.02 $0.06 \mathrm{~m} \mathrm{~s}^{-1}$, MaxError 3.4-7.1\% 1RM) and the preferred reference to compare with other technologies. OEC and LPT were the second-best alternatives (SDC $0.06-0.11 \mathrm{~m} \mathrm{~s}^{-1}$ ), always considering the particular margins of error for each exercise and velocity outcome. ACC and VBS are not recommended given their substantial errors and uncertainty of the measurements $\left(\mathrm{SDC}>0.13 \mathrm{~m} \mathrm{~s}^{-1}\right)$.
\end{abstract}

Keywords-Standard error of measurement, Velocity-based resistance training, Exercise testing, Monitoring, Strength performance, Validity.

Address correspondence to Jesús G. Pallarés, Human Performance and Sports Science Laboratory, Faculty of Sport Sciences, University of Murcia, C/ Argentina s/n, Santiago de la Ribera, Murcia, Spain. Electronic mail: jgpallares@um.es

\section{INTRODUCTION}

Considerable research attention has been paid to monitoring movement velocity during resistance training in recent years. ${ }^{14,15,26,30}$ Velocity-based resistance training (VBRT) has been proposed as an effective method to better characterize the resistance training stimulus and, specifically, to more precisely gauge the actual effort or intensity at which athletes train. VBRT requires the use of particular technologies to monitor bar velocity during training, and it has multiple practical applications. ${ }^{15,25,28,30-33}$ VBRT has been found to be a robust, non-invasive and highly sensitive method to estimate key performance indicators, such as the relative loading intensity, maximum strength (one-repetition maximum, 1RM) and the level of effort and neuromuscular fatigue incurred during a training set. ${ }^{15,22,25,28,31,32}$ These practical applications are however dependent on the actual degree of reliability exhibited by the different existing technologies and particular devices currently used for measuring bar velocity. It has been shown that small changes in the velocity developed against some reference workloads are accompanied by critical improvements in the neuromuscular and functional performance of well-trained athletes. For instance, an increment in mean concentric velocity of just 0.07 to $0.10 \mathrm{~m} \mathrm{~s}^{-1}$ is associated with improvements of $\sim 5 \% 1 \mathrm{RM}$ strength in main resistance exercises such as the bench press (BP), full back squat (SQ) and prone bench pull (PBP). ${ }^{15,22,31,32}$ Thus, in order to successfully implement a VBRT intervention, it is imperative to use sufficiently accurate and reliable technologies for measuring bar velocity. ${ }^{16}$ 